\title{
Correction to: First Genome of Labyrinthula sp., an Opportunistic Seagrass Pathogen, Reveals Novel Insight into Marine Protist Phylogeny, Ecology and CAZyme Cell-Wall Degradation
}

\author{
Mun Hua Tan ${ }^{1,2,3,4} \cdot$ Stella Loke ${ }^{1,2} \cdot$ Laurence J. Croft $^{1,2} \cdot$ Frank H. Gleason $^{5} \cdot$ Lene Lange $^{6} \cdot$ Bo Pilgaard $^{7}$. \\ Stacey M. Trevathan-Tackett ${ }^{1}$ (D)
}

Published online: 6 July 2021

๑) Springer Science+Business Media, LLC, part of Springer Nature 2021

\section{Correction to: Microbial Ecology}

https://doi.org/10.1007/s00248-020-01647-x

The supplementary materials contained errors. The original article has been corrected.

The original article can be found online at https://doi.org/10.1007/ s00248-020-01647-x.

Stacey M. Trevathan-Tackett

s.trevathantackett@deakin.edu.au

1 Centre of Integrative Ecology, School of Life and Environmental Sciences, Deakin University, Geelong, Victoria, Australia

2 Deakin Genomics Centre, Deakin University, Geelong, Victoria, Australia

3 School of BioSciences, Bio21 Institute, University of Melbourne, Parkville, Victoria, Australia

4 Department of Microbiology and Immunology, University of Melbourne, Bio21 Institute, Melbourne, Victoria, Australia

5 School of Life and Environmental Sciences, University of Sydney, Sydney, New South Wales, Australia

6 BioEconomy, Research \& Advisory, Valby, Copenhagen, Denmark

7 Protein Chemistry and Enzyme Technology, Department of Bioengineering, Technical University of Denmark, Kgs. Lyngby, Denmark 